\title{
Three-dimensional hybrid simulation of magnetized plasma flow around an obstacle
}

\author{
Hironori Shimazu \\ Space Science Division, Communications Research Laboratory, Koganei, Tokyo 184-8795, Japan
}

(Received February 16, 1999; Revised May 27, 1999; Accepted June 1, 1999)

\begin{abstract}
The interaction between the magnetized plasma flow and an obstacle was investigated in the computer simulations described here by using a three-dimensional hybrid code (kinetic ions and massless fluid electrons). The results, which are relevant to the interaction between the solar wind and an unmagnetized planet (Venus or Mars), show that fundamental structures (bow shock and magnetotail) are formed. When a reflecting boundary is used at the obstacle, the magnetic field configuration was clearly asymmetrical in the direction of the convection electric field. This asymmetry is a result of differences in ion acceleration due to the convection electric field. Asymmetry is also evident when the size of the obstacle is close to the Larmor radius of protons. The shock of a smaller obstacle is weaker than that of a larger obstacle, but the shock size is almost independent of the obstacle size.
\end{abstract}

\section{Introduction}

Magnetized plasma flow around an obstacle has been studied and the results of these studies have been applied to the interaction between the solar wind and unmagnetized planets (Venus and Mars). Since the solar wind is supersonic and the mean free path of the plasma particles constituting the solar wind is much larger than the size of planets, the collisionless shock is formed in front of the planet. The gas dynamic theory has been used to explain the location and shape of the shocks around Venus and Mars (Spreiter et al., 1970; Slavin et al., 1983). The solar wind interaction with these planets has also been the subject of magnetohydrodynamic (MHD) simulations. Tanaka (1993) performed threedimensional MHD simulations of the interaction between the solar wind and an unmagnetized planet. He investigated the configuration of the magnetic field around the planet and also explained the magnetic field draping process.

Although the gas dynamic theory and MHD simulations have been useful in investigating many kinds of problems, they are invalid when the scale of the Larmor radius of ions must be considered. Since the radius of Mars, for example, is comparable to the Larmor radius of solar wind protons, the solar wind interaction with Mars may differ from that with Venus. When we investigate the solar wind interaction with Mars, we must therefore use some other methods taking into account the effect of the finite Larmor radius of protons.

The hybrid code is one of the best simulation methods for studying the effect because it treats ions as gyrating particles (Leroy et al., 1982). Brecht (1990) and Brecht et al. (1993) used a hybrid code in their three-dimensional simulations of the interaction between the solar wind and the dayside portion of Mars. They showed that the large Larmor radius of the solar wind ions prevented the formation of a traditional

Copy right (c) The Society of Geomagnetism and Earth, Planetary and Space Sciences (SGEPSS); The Seismological Society of Japan; The Volcanological Society of Japan; The Geodetic Society of Japan; The Japanese Society for Planetary Sciences. collisionless shock in the subsolar region of the interaction. Moore et al. (1991) also used three-dimensional hybrid code simulations of the interaction between the solar wind and the dayside portion of an unmagnetized planet, and they examined the asymmetry in the direction of the convection electric field. Brecht (1997) included the magnetotail region in his simulation box and showed that the resultant magnetic field configuration around Mars is consistent with observations.

This paper reports the results of three-dimensional hybrid code simulations in a global simulation system (size of the simulation box $\gg$ size of the obstacle) including the magnetotail. The simulations were similar to that of Brecht (1997) in that they included the magnetotail, but the inner boundary condition at the obstacle was different. He used an absorbing boundary, whereas the simulations reported here used a reflecting boundary.

The treatment of the boundary condition at the obstacle is an important problem, and to treat the boundary rigorously we would need to simulate a plasma representing the ionosphere around the planet (Shimazu et al., 1996). To avoid this complication and to make the underlying physical processes easier to understand, some simple boundary conditions have been used instead. In the simulations reported by Moore et al. (1991) and by Brecht (1997), ions were assumed to be absorbed at the obstacle: the entire mass flux was assumed to be absorbed.

The reflecting boundary, however, in which there is no mass flux into the obstacle, should also be examined. Gas dynamic calculations have assumed that the flow lines do not connect with the obstacle: the normal component of the flow velocity was set to zero at the surface of the obstacle. This condition in MHD models corresponds to the reflecting boundary condition in particle simulations, where the normal component of the flow velocity should be zero as a result of the pressure balance. Because the distribution function is symmetric in the direction normal to the reflect- 
ing boundary, the average mass flux between the solar wind and the obstacle becomes zero. Particle simulations with a reflecting boundary are, thus, a natural extension of MHD simulations and are more appropriate for comparisons with the gas dynamic theory or MHD simulations than are particle simulations with an absorbing boundary. Disadvantages of using the reflecting boundary, however, are that the reflected ions might change the distribution function and consequently result in the generation of some plasma instabilities.

The reflecting boundary was used in the present work for the following reason: Consider the problem posed by the trajectories of shocked (decelerated and heated) solar wind ions that penetrate the ionosphere. If these ions reach a depth where collisions with atmospheric neutrals species must be considered, the lower ionosphere will rapidly gain a net charge. Electrons, which have a smaller Larmor radius, cannot penetrate as deeply as ions. As a result, charges are separated and an electric field is generated. This electric field either prevents ions from entering the ionosphere or decreases the depth to which they penetrate. Consequently, solar wind ions that penetrate the ionosphere return to the flow region again.

The present simulations did not include the effects of charge exchange, ion pickup, or mass loading, even though the importance of these effects is well known (Intriligator, 1982; Luhmann et al., 1985; Phillips et al., 1987). To understand the importance of these effects we first need to exclude them from the simulation. The focus of this study was the effect of the finite Larmor radius of protons on the asymmetry of the bow shock, magnetosheath, and magnetotail.

\section{Model}

The simulation used the three-dimensional Cartesian coordinate system in which the grid cells were spaced equally and there were 32 in each of the $x, y$, and $z$ directions. The average number of particles in one grid cell was 16 . The initial conditions are shown in Fig. 1. The solar wind was emulated by using a super-Alfvénic plasma (number density $n_{0}$; velocity $\boldsymbol{v}_{\mathrm{sw}}$ ) continuously injected into the simulation system from the $x=0$ plane (left-hand boundary of Fig. 1). Although the left-hand side of the simulation box was a reflecting boundary, few ions were reflected there because $v_{\mathrm{sw}}$ was initially set to be greater than the ion thermal velocity. The bulk velocity of the solar wind was parallel to the $x$ axis, and solar wind ions were removed from the simulation system when they reached the right-hand boundary. The only ions considered in the work reported here were protons. The sphere that represents an obstacle was placed at the center of the simulation box, and the radius $R$ of the obstacle was sufficiently smaller than the size $L$ of the simulation box $(L=8 R)$ that influences of the boundaries of the simulation box were insignificant.

Two kinds of boundary conditions for ions at the obstacle were considered: absorption and reflection. The condition for absorption was simple: any ion that moved into the obstacle was removed from the simulation system. The condition for reflection can be explained using Fig. 1. Consider the point $\mathrm{P}_{0}$, which is the location of an ion at a certain time. If the ion moved into the obstacle to point $\mathrm{P}_{1}$ at the next time step, it was reflected to $\mathrm{P}_{2}$, the mirror point of $\mathrm{P}_{1}$ with regard

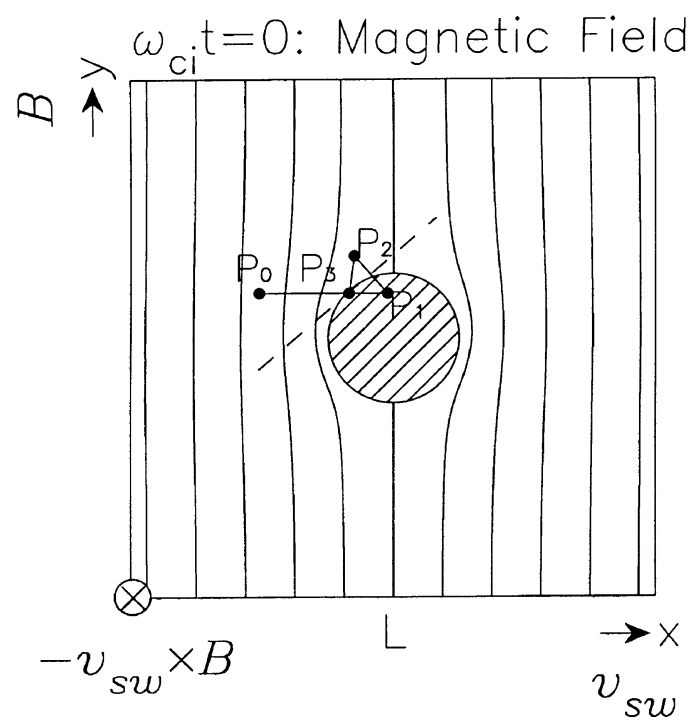

Fig. 1. Initial conditions. This figure is a cross section on the $z=L / 2$ plane, which includes the center of the obstacle (represented by the circle). The solid lines represent the initial ambient magnetic field lines.

to the tangent plane including $\mathrm{P}_{3}$. The velocity vector was also transformed into its mirror-image with respect to this tangent plane. Momentum was the same before and after the reflection.

The initial ambient magnetic field $\boldsymbol{B}$ (shown by lines in Fig. 1) was given by the potential field:

$$
\boldsymbol{B}= \begin{cases}\nabla\left\{B_{0} y\left(1+\frac{R^{3}}{2 r^{3}}\right)\right\} & (r>R) \\ 0 & (r<R),\end{cases}
$$

where $B_{0} /\left(4 \pi m_{\mathrm{i}} n_{0}\right)^{1 / 2} \equiv V_{\mathrm{A}}=1.0 \times 10^{-4} c$ and $r^{2}=$ $(x-L / 2)^{2}+(y-L / 2)^{2}+(z-L / 2)^{2}$. Because the electrical resistivity of the solar wind was assumed to be zero the formation of the bow shock was due to the numerical resistivity. This resistivity does not affect the intensity, shape, or location of the bow shock. The resistivity $\eta$ of the obstacle was given by

$$
\eta \omega_{\mathrm{ci}} / 4 \pi=1.0 \times 10^{-8},
$$

where $\omega_{\mathrm{ci}}\left(=e B_{0} /\left(m_{\mathrm{i}} c\right)\right)$ is the proton cyclotron frequency. This value corresponds to the resistivity of the lower ionosphere. When the electric and magnetic fields were calculated, the fields in the interior of the obstacle were also calculated, but the electron velocity there was set to zero.

The initial temperature of the protons was assumed to be the same as that of the electron fluid and to be constant in space. The ratio of the proton thermal pressure to the magnetic pressure of the solar wind (i.e., $\beta_{\mathrm{i}}$ ) was assumed in this study to be 1 , whereas the previous simulations had assumed that the ions were cold. The results of simulations with $\beta_{\mathrm{i}}=0.5$ and $\beta_{\mathrm{i}}=2$ showed that variations of $\beta_{\mathrm{i}}$ within this range did not change the physics drastically. Slavin and Holzer (1981) showed that $\beta$ ( $=\beta_{\mathrm{i}}+\beta_{\mathrm{e}}, \beta_{\mathrm{e}}$ is $\beta$ for electrons) is 1.4 at Venus and is 2.0 at Mars. That $\beta_{\mathrm{i}}$ equals 1 is thus a reasonable assumption for the solar wind in this study. 

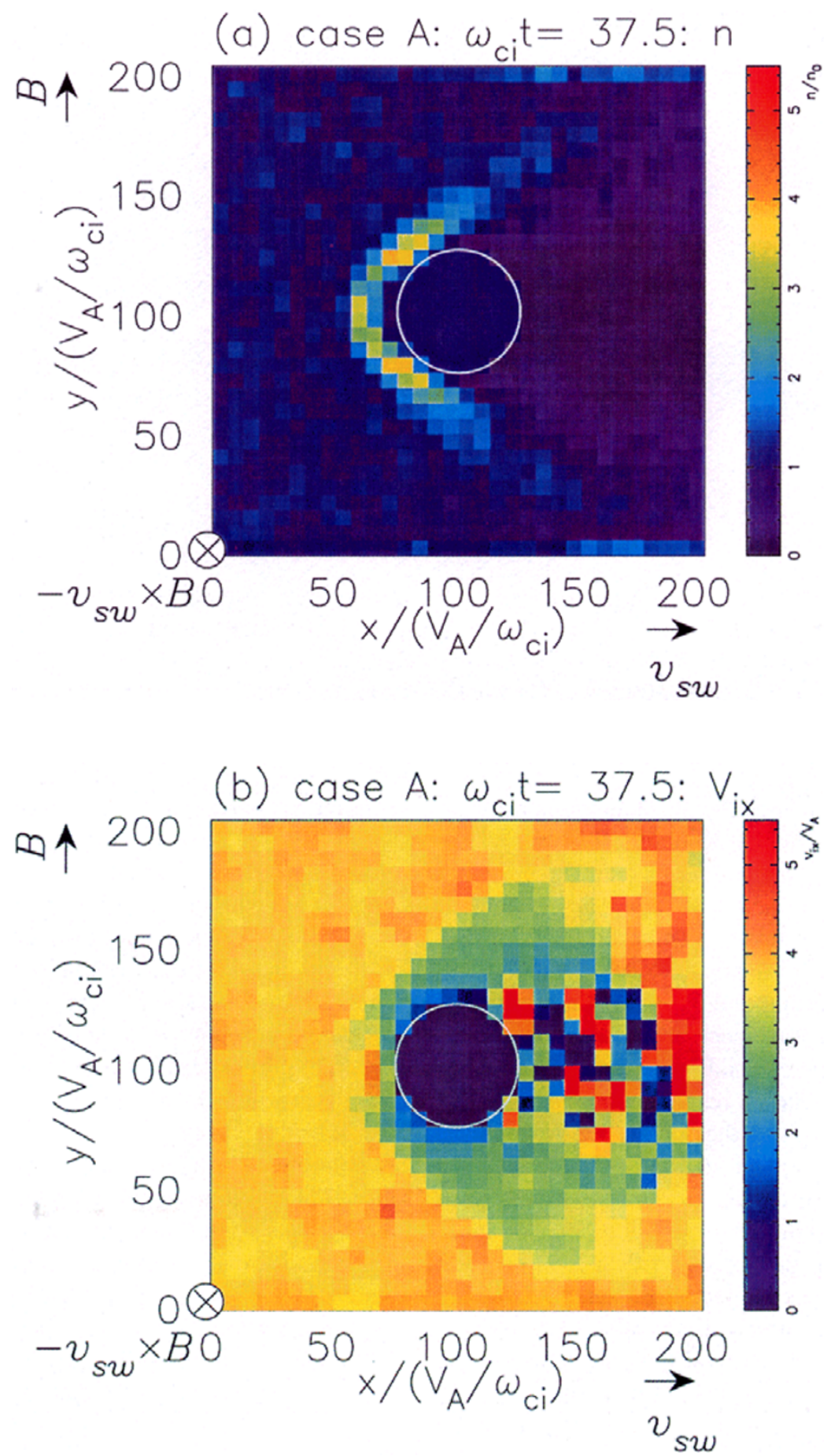

Fig. 2. The $z=L / 2$ plane at $\omega_{\mathrm{ci}} t=37.5$ in case A: (a) proton density and (b) the $x$ component of the proton velocity. The center circle represents the obstacle.

\section{Results}

\subsection{Asymmetry}

This subsection describes the fundamental structures obtained in the simulation. They must be examined in detail before they are compared with the structures described in later sections. The results described here are those obtained in what is called case $\mathrm{A}$ in this paper. In this case the radius of the obstacle was set to $25.6 V_{\mathrm{A}} / \omega_{\mathrm{ci}}$. Since $v_{\mathrm{sw}}$ was fixed at $4.0 V_{\mathrm{A}}$, the average Larmor radius $\rho$ of protons in the simulation is given by

$$
\rho=v_{\mathrm{sw}} / \omega_{\mathrm{ci}}=4 V_{\mathrm{A}} / \omega_{\mathrm{ci}} .
$$

The radius $R$ of the obstacle was thus about $6.4 \rho$. The time step was $\omega_{\mathrm{ci}} \Delta t=0.025$. Various mesh sizes and time steps were tested in preliminary simulations, and the results reported here were obtained with an obstacle diameter of 8 cells providing physically meaningful results.

Figures 2(a) and 2(b) respectively show the proton density and the $x$ component of the average proton velocity at $\omega_{\mathrm{ci}} t=37.5$. During the time $37.5 / \omega_{\mathrm{ci}}$ the initial solar wind travels a distance $150 V_{\mathrm{A}} / \omega_{\text {ci }}$ or about $0.73 \mathrm{~L}$. As shown in 


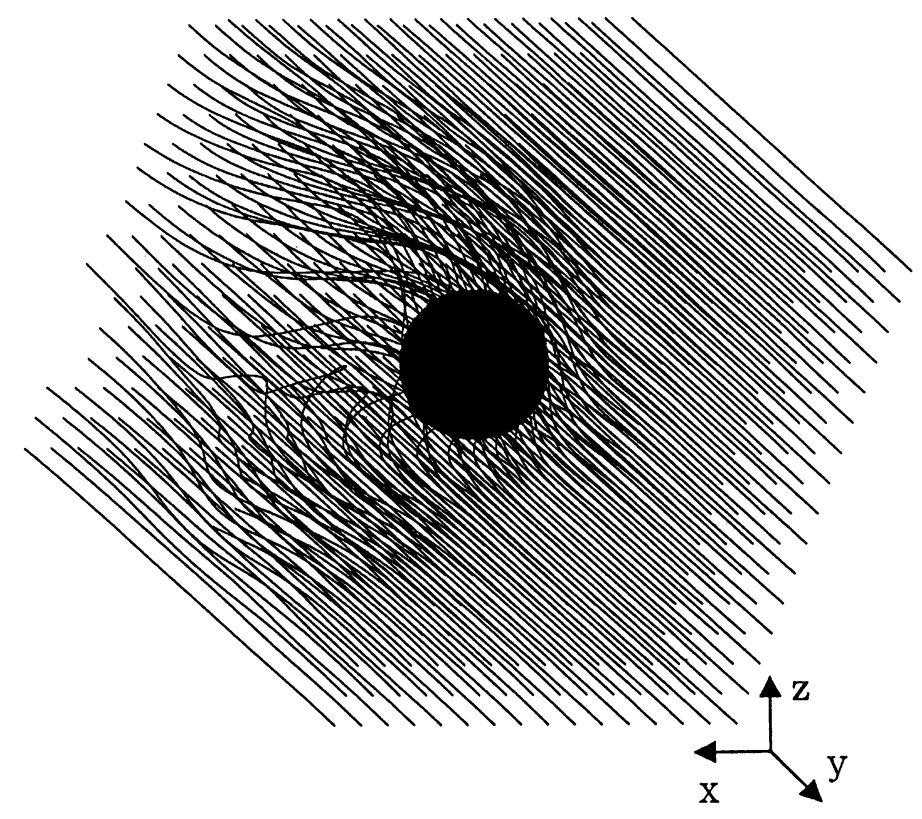

Fig. 3. Magnetic field lines obtained at $\omega_{\mathrm{ci}} t=37.5$ in case A.

Fig. 2, on the upstream side of the obstacle the density increases sharply and the velocity decreases sharply. A bow shock is generated there. The total pressure on the upstream side is given by the initial conditions: (dynamic pressure) + (thermal pressure (ion+electron $))+($ magnetic pressure $)=$ $16.0 n_{0} k_{\mathrm{B}} T_{\mathrm{e}}+2.0 n_{0} k_{\mathrm{B}} T_{\mathrm{e}}+1.0 n_{0} k_{\mathrm{B}} T_{\mathrm{e}}=19.0 n_{0} k_{\mathrm{B}} T_{\mathrm{e}}$, where $k_{\mathrm{B}}$ is the Boltzmann constant and $T_{\mathrm{e}}$ is the temperature of the electron fluid (constant in space and time). And the total pressure on the downstream side near the subsolar point is obtained from the simulation results: the density on the downstream side is 2.6 times that on the upstream side, the velocity on the downstream side is 0.39 times that on the upstream side, the ion temperature on the downstream side is 2.4 times that on the upstream side, and the magnetic field intensity on the downstream side is 2.8 times that on the upstream side. Thus (dynamic pressure) + (thermal pressure $)+($ magnetic pressure $)=6.3 n_{0} k_{\mathrm{B}} T_{\mathrm{e}}+3.4 n_{0} k_{\mathrm{B}} T_{\mathrm{e}}+$ $7.8 n_{0} k_{\mathrm{B}} T_{\mathrm{e}}=17.5 n_{0} k_{\mathrm{B}} T_{\mathrm{e}}$. The total pressure is nearly the same on both sides of the shock ( $8 \%$ difference).

The magnetotail is formed on the downstream side of the obstacle. The velocity field is disturbed in the tail because the density there is very low. Since this simulation used a filter to reduce short-wavelength electromagnetic fields in the tail region, the tail region may be rather diffusive.

Figure 3 shows the magnetic field lines around the obstacle at $\omega_{\mathrm{ci}} t=37.5$. The field lines are bent where the solar wind encounters the shock, and the magnetic field piles up downstream of the shock.

Four kinds of asymmetry in the direction of the $-\boldsymbol{v}_{\mathrm{sw}} \times \boldsymbol{B}$ convection electric field ( $-z$ direction) were found in this case:

3.1.1 Proton acceleration Figure 4(a) shows, for the $y=L / 2$ plane, the $x$ component of the proton velocity. The flow near $x=100 V_{\mathrm{A}} / \omega_{\mathrm{ci}}, z=70 V_{\mathrm{A}} / \omega_{\mathrm{ci}}$ (near the "pole" on the side of the obstacle to which the convection electric field is pointing) is accelerated to a speed greater than that of the solar wind, while the flow on the other side is not. This is a clear asymmetry in the direction of the convection electric field. As shown in Fig. 4(b), however, the flux $n v_{\text {ix }}$ in this acceleration region is very low because the density there is very low. This proton acceleration was not observed in previous simulation studies using an absorbing boundary, This acceleration is considered in detail in the next subsection.

3.1.2 Shock size We can see in Fig. 4 that the distance from the center of the obstacle to the shock is shorter on the side of the obstacle to which the convection electric field is pointing than that on the other side. Because the fast mode Mach cone asymmetry does not appear in this direction, the asymmetry in shock size must be a kinetic effect. This asymmetry in shock size is consistent with the simulation results of Brecht and Ferrante (1991), but is opposite the asymmetry actually observed at both Venus (Alexander et al., 1986; Russell et al., 1988) and Mars (Zhang et al., 1991). This discrepancy indicates that the observed asymmetry is not related to the effect of the finite Larmor radius and must thus be due to effects not included in the simulations.

Brecht and Ferrante (1991) concluded that the asymmetry in shock size was caused by the Hall current. In the present model, however, it is a result of the asymmetry in ion acceleration: on the side of the obstacle to which the convection electric field is pointing, ions are accelerated and less accumulated as explained in detail in the following subsection. The asymmetry can be accounted for by the Hall current (since the ion acceleration causes the ion current to differ from the electron current), but the present results show that what is essential to the asymmetry is the asymmetry of the ion acceleration.

3.1.3 Magnetic field configuration in the magnetotail Figure 3 shows the field lines draping around the obstacle and forming the magnetotail. The magnetic field configuration 

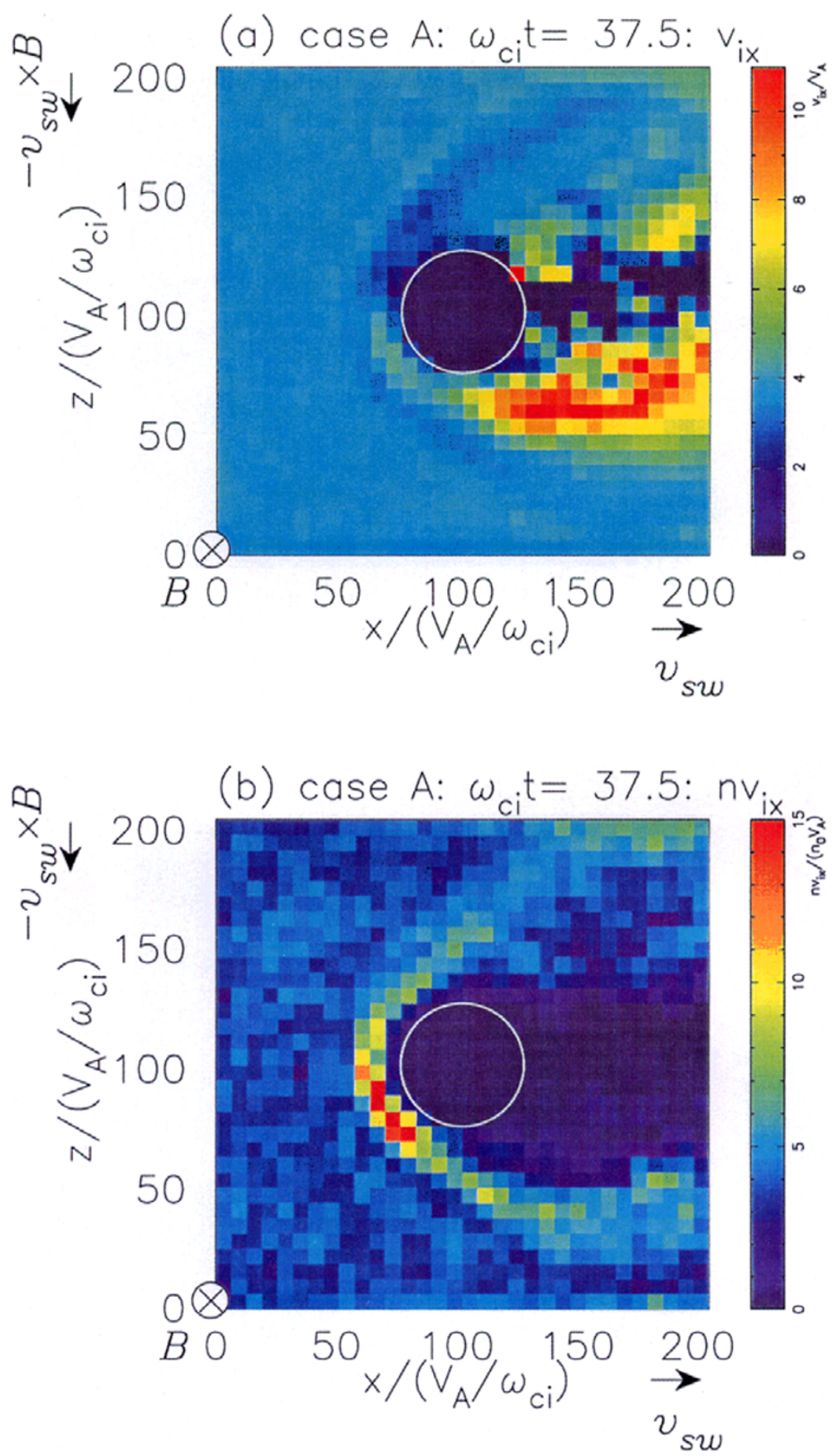

Fig. 4. The $y=L / 2$ plane at $\omega_{\mathrm{ci}} t=37.5$ in case A: (a) the $x$ component of the proton velocity and (b) the flux $n v_{\mathrm{ix}}$.

in the magnetotail is obviously asymmetrical in the $z$ direction: the field lines in the negative $z$ direction (direction of the convection electric field) are bent more than those in the positive $z$ direction, and the tail is filled with field lines originating on the negative $z$ side. The previous studies reported asymmetry of the magnetic field only in the dayside (Moore et al., 1991; Brecht et al., 1993). In the present results the nightside asymmetry is also shown. This asymmetric filling of magnetic fields in the magnetotail was suggested by Slavin et al. (1989) on the basis of PVO observations, suggesting the interplanetary flux tubes bent into the tail predominantly over the hemisphere to which the convection electric field was pointing. This asymmetry too is a result of the asymmetry of ion acceleration as shown later in this paper.

3.1.4 Field line draping Figure 5(a) shows, for the $x=(5 / 8) L$ plane in the tail, the $x$ component of the magnetic field. The sign of the $B_{x}$ component is positive in the right half of the figure and negative in the left half, and this figure shows the clear two-lobe structure in the magnetotail. This structure is characteristic of the draped field lines in the 

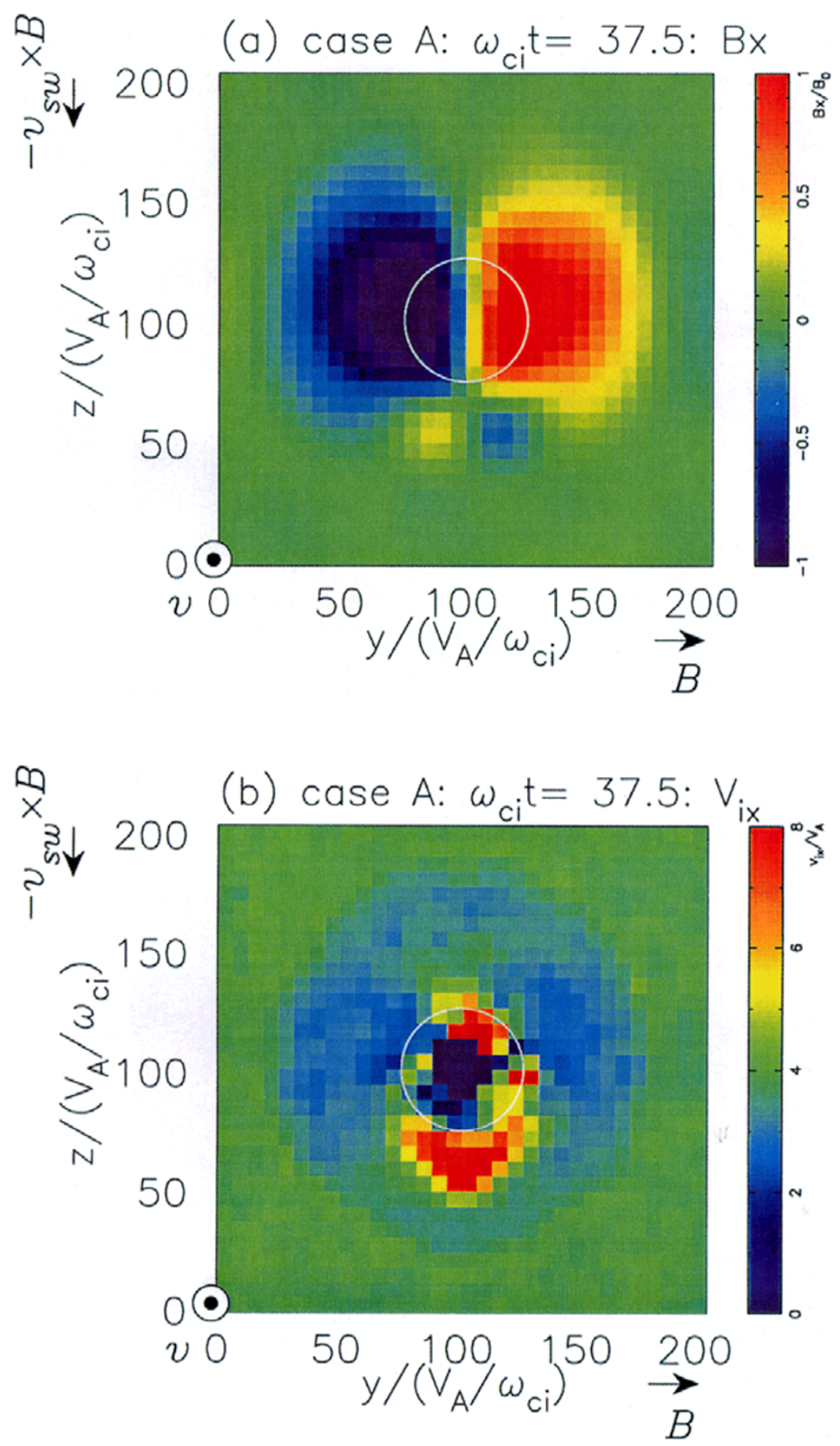

Fig. 5. The $z=(5 / 8) L$ plane at $\omega_{\mathrm{ci}} t=37.5$ in case A: (a) the $x$ component of the magnetic field and (b) the $x$ component of the proton velocity.

induced magnetotail.

Marubashi et al. (1985) and Saunders and Russell (1986) used the PVO data when they analyzed the interplanetary origin of the Venus magnetotail, and they found that the positive and negative polarity of the $B_{x}$ component was clearly divided into two parts. Yeroshenko et al. (1990), using the Phobos 2 data, showed the same pattern of magnetic field polarity in the Mars magnetotail. The clear two-lobe structure obtained in the present simulation results is in good agreement with the observation results.

Figure 5(b) shows, also for the $x=(5 / 8) L$ plane, the $x$ component of the proton velocity. The inner circle shows the obstacle, and the outer milled one corresponds to the shock. The solar wind flow decelerates inside the shock, and a fast proton flow is noticeable near $y=100 V_{\mathrm{A}} / \omega_{\mathrm{ci}}, z=$ $70 V_{\mathrm{A}} / \omega_{\mathrm{ci}}$. This fast flow is seen only on the side of the obstacle to which the convection electric field is pointing. It is notable from Fig. 5(a) that the signs of the $B_{x}$ component in the fast flow region are the opposite of those in the other part. In this region the direction of the magnetic field is opposite that of the draped magnetotail field. This inverse draping polarity of the magnetic field suggests that the fast 


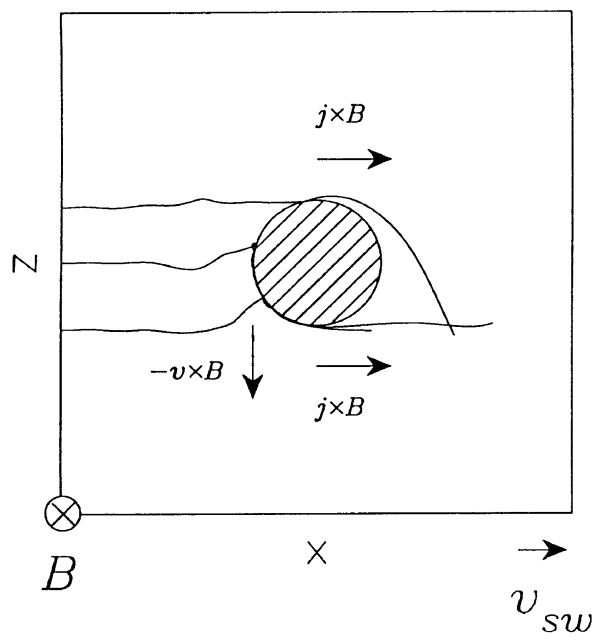

Fig. 6. For the $y=L / 2$ plane in case A, three proton trajectories. The center circle represents the obstacle.

flow drags the field lines.

\subsection{Proton acceleration}

Figure 6 shows three proton trajectories obtained in case A. As shown in Fig. 4(a), protons were accelerated near the "pole" on the side of the obstacle to which the convection electric field was pointing. Two factors responsible for this acceleration were investigated in these simulations.

The first is the proton reflection at the obstacle. Note that here we are considering protons reflected at the obstacle and are neglecting the reflection at the shock. Protons reflected from the side of the obstacle to which the $-\boldsymbol{v}_{\mathrm{sw}} \times \boldsymbol{B}$ convection electric field is pointing should be accelerated because the direction in which they are reflected is that to which the convection electric field is pointing.

This acceleration should be proportional to the solar wind magnetic field $B$ and $v_{\mathrm{sw}}$. This expectation was examined by changing the simulation parameters. Figure 7(a) shows the relation between $B$ and $\Delta v_{1}$, which is the difference between the shocked solar wind velocity and the velocity measured at the "pole" (the side of the obstacle to which the convection electric field is pointing). This figure shows that $\Delta v_{1}$ is roughly proportional to $B$. Moreover, when the solar wind velocity was set to $2 V_{\mathrm{A}}, \Delta v_{1}$ became nearly half.

The second factor responsible for the proton acceleration is the $\boldsymbol{j} \times \boldsymbol{B}$ force (magnetic tension). The magnitude of the acceleration due to this force becomes large on both flanks of the obstacle near $x=110 V_{\mathrm{A}} / \omega_{\mathrm{ci}}, z=70 V_{\mathrm{A}} / \omega_{\mathrm{ci}}$, and $z=130 V_{\mathrm{A}} / \omega_{\mathrm{ci}}$. The measured value of the acceleration is $|\boldsymbol{j} \times \boldsymbol{B}| /\left(m_{\mathrm{i}} n c\right)=0.3 V_{\mathrm{A}} \omega_{\mathrm{ci}}$, and the extent of the region over which this acceleration acts is $50 V_{\mathrm{A}} / \omega_{\mathrm{ci}}$. If we use these values to estimate the proton velocity, while assuming the acceleration is constant, the final velocity becomes $v=$ $5.6 V_{\mathrm{A}}$ when the initial velocity is $V_{\mathrm{A}}$. This initial velocity corresponds to the proton velocity on the side opposite the one to which the convection electric field is pointing. On the other hand, since protons on the side to which the convection electric field is pointing have already been accelerated, the initial average velocity on this side is taken to be $4.0 V_{\mathrm{A}}$. Then $v$ becomes $6.8 V_{\mathrm{A}}$. These average velocities can account for
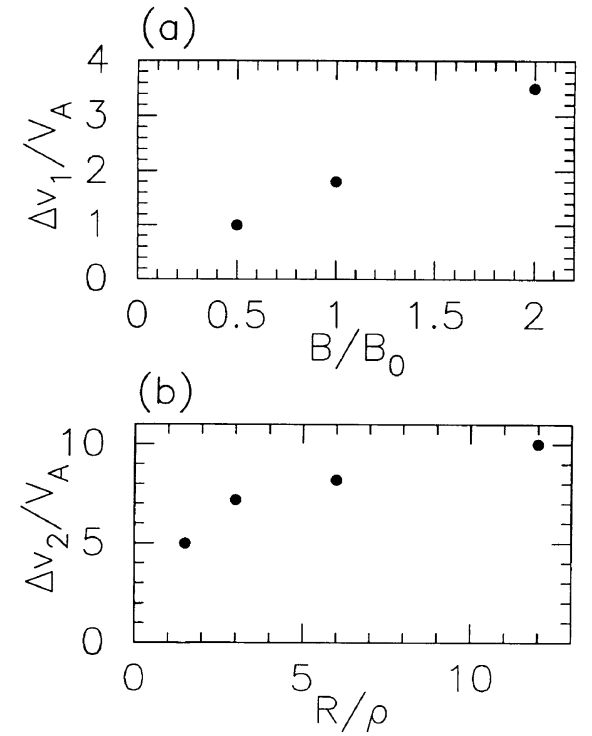

Fig. 7. (a) Relation between $\Delta v_{1}$ and $B$ when $R$ is $25.6 V_{\mathrm{A}} / \omega_{\mathrm{ci}}$ and $v_{\mathrm{SW}}$ is $4 V_{\mathrm{A}}$. (b) Relation between $\Delta v_{2}$ and $R$ when $v_{\mathrm{sw}}$ is $4 V_{\mathrm{A}}$ and $B$ is $B_{0}$.

the simulation results.

If this second factor were responsible for the acceleration, the velocity difference $\Delta v_{2}$ should be given by

$$
\Delta v_{2} \sim|\boldsymbol{j} \times \boldsymbol{B}| \delta t \sim \frac{B^{2}}{l} \delta t,
$$

where $\delta t$ is the length of time the protons stay in the acceleration region and $l$ is the typical length of bent field lines. It was assumed that $l \sim R$, and $\delta t$ was taken to be the magnetic diffusion time of the obstacle $\left(\sim R^{2} / \eta\right)$ because the length of the stretched field lines would be proportional to the diffusion time. Thus,

$$
\Delta v_{2} \sim \frac{B^{2} R}{\eta} .
$$

Figure 7(b) shows the relation between $R$ and $\Delta v_{2}$, which is the difference between the velocity at the "pole" and that in the tail region. Although $\Delta v_{2}$ increases when $R$ increases, the increases are not proportional. It may not be appropriate to have assumed that $\delta t$ is the diffusion time because some of the field lines bend at the surface and do not penetrate the obstacle. Moreover, cases in which $B$ was set to $2 B_{0}$ and $0.5 B_{0}$ were examined, as were cases in which $\eta$ was set to $0.1 \eta$ and $0.01 \eta$. Although the acceleration increased with increasing $B$ and with decreasing $\eta$, the measured relation did not satisfy the relation (5), which may also be due to the slipping field lines.

The former section showed that the accelerated flow drags the field lines in the acceleration region. In this region protons had been accelerated too fast and the $\boldsymbol{j} \times \boldsymbol{B}$ force decelerated the flow rather than accelerated it.

We examined dependence on $\beta$, but the results are not so different. Thus the electron pressure term does not affect the acceleration.

Tanaka (1993) showed in his MHD simulation results that the tailward flow in the magnetosheath over the pole can be 

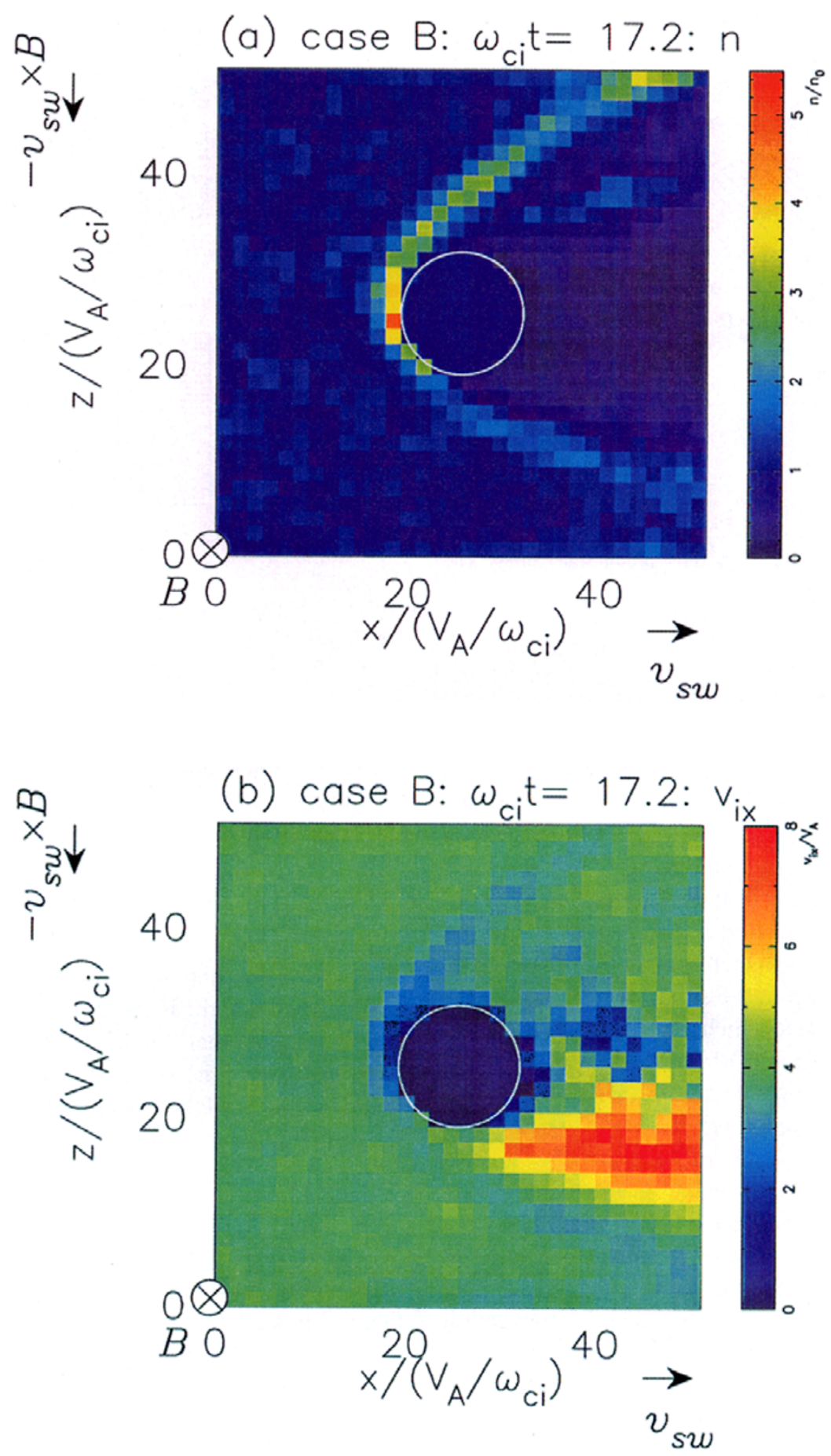

Fig. 8. The $y=L / 2$ plane at $\omega_{\mathrm{ci}} t=17.2$ in case B: (a) proton density and (b) the $x$ component of the proton velocity.

faster than the solar wind because of the $\boldsymbol{j} \times \boldsymbol{B}$ force. The present simulation showed the same results and also revealed that the $-\boldsymbol{v}_{\mathrm{sw}} \times \boldsymbol{B}$ electric field is important.

\subsection{Small obstacle}

This subsection shows the simulation results obtained when the obstacle radius $R$ was $6.4 V_{\mathrm{A}} / \omega_{\mathrm{ci}}$ and the time step was $\omega_{\mathrm{ci}} \Delta t=0.003125$. Here $\rho$ was $1.6 R$, and the boundary was assumed to be a reflecting boundary. This case is called case B in this paper. Figures 8 (a) and 8(b) respectively show the proton density and the $x$ component of the proton velocity for the $y=L / 2$ plane at $\omega_{\mathrm{ci}} t=17.2$. The initial solar wind travels a distance $69 V_{\mathrm{A}} / \omega_{\mathrm{ci}}>L$ during the time $17.2 / \omega_{\mathrm{ci}}$. The proton acceleration near the "pole" on the side of the obstacle to which the convection electric field is pointing is also seen in this case (Fig. 8(b)), as is the asymmetry of the acceleration. This study did not find that the asymmetry is more pronounced for a smaller obstacle, and in this respect is consistent with Brecht (1990).

The ratio of downstream density to upstream density of the shock is smaller in case $\mathrm{B}(\sim 2.2)$ than in case $\mathrm{A}(\sim 2.6)$, so 

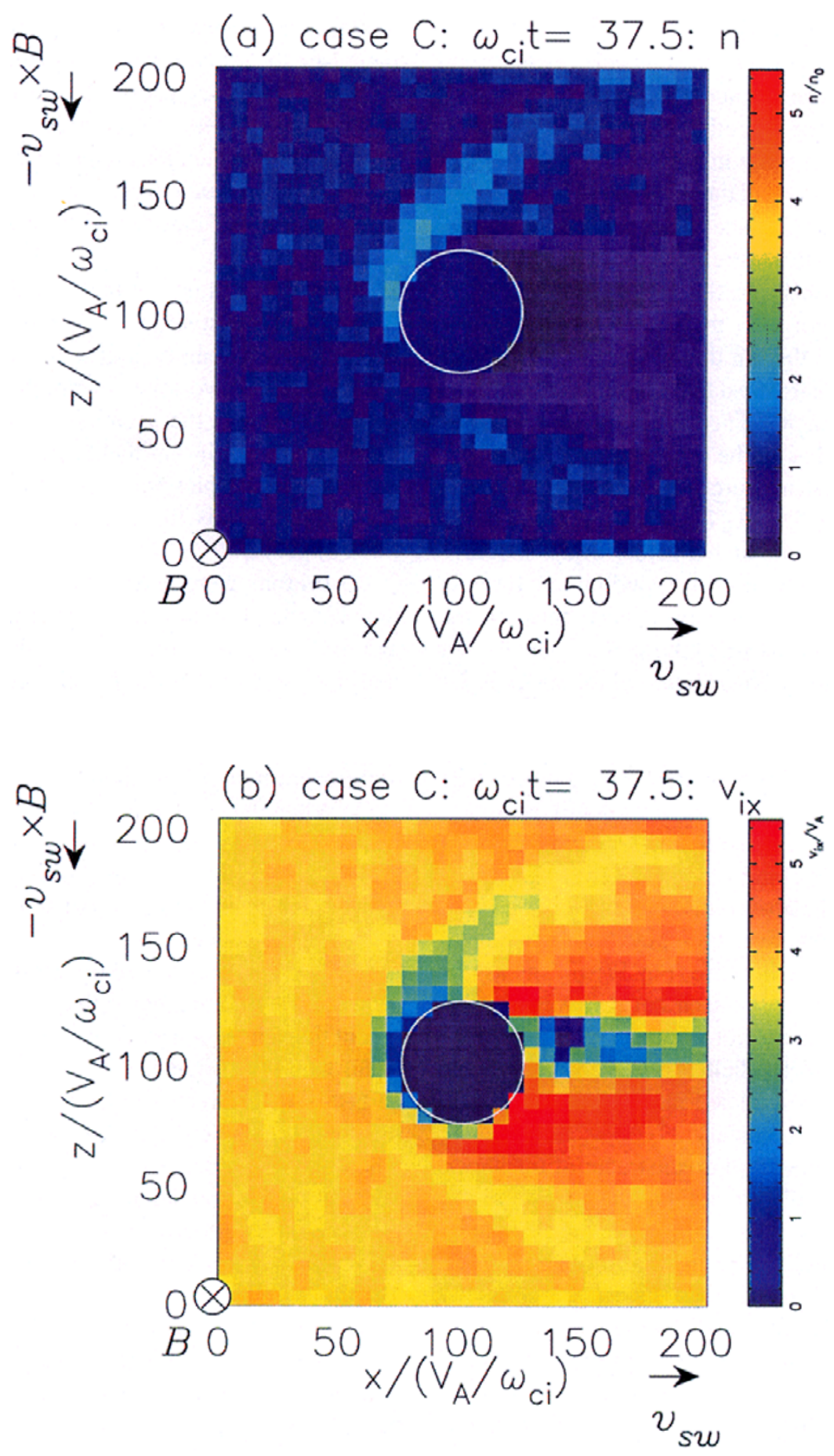

Fig. 9. The $y=L / 2$ plane at $\omega_{\mathrm{ci}} t=31.3$ in case C: (a) proton density and (b) the $x$ component of the proton velocity.

the shock in case B is weaker than that in case A. This shock is weaker because protons, which have a relatively large Larmor radius, rapidly move tailward and do not accumulate downstream of the shock. The total pressure on the downstream side is obtained from the present results: the density on the downstream side is 2.2 times that on the upstream side, the velocity on the downstream side is 0.48 times that on the upstream side, the ion temperature on the downstream side is 1.5 times that on the upstream side, and the magnetic field intensity on the downstream side is 2.5 times that on the upstream side. Thus (dynamic pressure) + (thermal pressure $)+($ magnetic pressure $)=8.1 n_{0} k_{\mathrm{B}} T_{\mathrm{e}}+2.5 n_{0} k_{\mathrm{B}} T_{\mathrm{e}}+$ $6.3 n_{0} k_{\mathrm{B}} T_{\mathrm{e}}=16.9 n_{0} k_{\mathrm{B}} T_{\mathrm{e}}$. The total pressure is nearly the same on both sides of the shock.

The ratio of the shock size to the obstacle size is smaller in case $\mathrm{B}(\sim 2.1)$ than in case $\mathrm{A}(\sim 2.3)$, but the difference is small. When the shock size dependence on obstacle size was examined by changing the obstacle size, the dependence was found to be weak. The observational results also did not show clear size dependence because the ratio for Mars is 
nearly the same as that for Venus (Slavin et al., 1983).

\subsection{Boundary condition}

This section shows the effect of the obstacle boundary by analyzing a case of absorbing boundary (here called case C) and comparing it with case A (reflecting boundary). Other parameters are the same as those in case A. Figures 9(a) and 9(b) show the proton density and the $x$ component of the proton velocity for the $y=L / 2$ plane at $\omega_{\mathrm{ci}} t=31.3$ in case $\mathrm{C}$. The total pressure on the downstream side is obtained from the present results: the density on the downstream side is 2.1 times that on the upstream side, the velocity on the downstream side is 0.48 times that on the upstream side, the ion temperature on the downstream side is 3.5 times that on the upstream side, and the magnetic field intensity on the downstream side is 2.3 times that on the upstream side. Thus (dynamic pressure $)+($ thermal pressure $)+($ magnetic pressure $)=$ $7.7 n_{0} k_{\mathrm{B}} T_{\mathrm{e}}+4.5 n_{0} k_{\mathrm{B}} T_{\mathrm{e}}+5.3 n_{0} k_{\mathrm{B}} T_{\mathrm{e}}=17.5 n_{0} k_{\mathrm{B}} T_{\mathrm{e}}$. The total pressure is nearly the same on both sides of the shock.

If we compare Fig. 9 with the corresponding one for case A, we can see that the difference in density between the regions upstream and downstream of the shock is smaller for case $\mathrm{C}$. In case $\mathrm{C}$ the density downstream of the shock is 2.1 times that in the solar wind, while in case $\mathrm{A}$ it is 2.6 times that in the solar wind.

This difference is due to ions being removed at the absorbing boundary. Moreover, the downstream velocity is greater in case $C$ than in case $A$ because the mass flux is not zero at the surface of the absorbing obstacle. Thus the shock becomes weaker under the absorbing boundary condition.

The acceleration regions near the "pole" are evident on both sides of the obstacle in this case (Fig. 9(b)). The asymmetry of the acceleration can be seen but it is weaker than that seen in case A. Because protons are not reflected, the effect of the convection electric field is relatively small. This case is similar to the cases modeled in previous simulation studies using an absorbing boundary.

The shock is located one mesh downstream of the shock in case A. Slavin et al. (1979) found that the altitude of the Venus bow shock varies with the solar cycle, being greatest at the solar maximum and lowest at the minimum. They argued that the enhanced neutral atmosphere scale heights at the cycle minimum lowered the bow shock altitude by increasing the charge exchange in the lower magnetosheath and thus increasing solar wind "absorption". Although the effect of this charge exchange was not explicitly included in the present simulation, the results show that the absorbing condition lowers the shock altitude.

When we consider this weaker and lower shock from the MHD viewpoint, 'weaker' and 'lower' seem to be conflicting. To resolve this apparent inconsistency, we should note that when the absorbing boundary is used, the effective size of the obstacle is smaller than it is when the reflecting boundary is used.

\section{Conclusions}

The present three-dimensional hybrid code simulations of the interaction between the solar wind and an unmagnetized planet considered a global and self-consistent simulation system that included the magnetotail. This simulation was also the first to use the reflecting boundary condition at the obstacle.

Differences between the previous models and the present model are the reflecting boundary and finite ion $\beta$ value. The reflecting boundary caused a tailward ion acceleration that had not been evident in previous simulation studies. Previous studies assumed ions to be cold, while in this simulation the $\beta$ value for solar wind ions was assumed to be 1 . This difference, however, seemed to have little effect on the results.

The results can be summarized as follows:

(1) The present simulations reproduced the fundamental structures: bow shock and magnetotail. They also reproduced the clear two-lobe tail structure observed by the PVO, and they showed the asymmetric filling of magnetic fields in the magnetotail that had been suggested by Slavin et al. (1989), but that had not been reported in previous simulation studies. This filling is evident when the boundary is reflecting.

(2) Protons were accelerated in the magnetosheath near the obstacle. Two kinds of acceleration processes were considered: one due to the $-\boldsymbol{v}_{\mathrm{sw}} \times \boldsymbol{B}$ convection electric field and the other due to the $\boldsymbol{j} \times \boldsymbol{B}$ force. When the reflecting boundary at the obstacle was used, the proton acceleration due to $-\boldsymbol{v}_{\mathrm{sw}} \times \boldsymbol{B}$ field was dominant. This acceleration led to the asymmetry of the magnetic field around the obstacle.

(3) When the size of the obstacle was close to the Larmor radius of protons, the shock became weak. This weak shock was a result of protons, which have a relatively large Larmor radius, rapidly moving tailward and not accumulating downstream of the shock.

(4) Two kinds of boundary conditions for protons at the obstacle were compared: reflection and absorption. The effect of the convection electric field was found to be relatively small when the boundary is absorbing. When ions are absorbed, they are removed from the downstream region and the downstream density therefore becomes smaller than it is when they are reflected. The downstream velocity is larger in the absorbing boundary case because the mass flux at the surface of the obstacle is not zero. Thus an absorbing boundary results in a weaker shock than does a reflecting boundary. The shock under the absorbing boundary was also found to be farther downstream than it was under the reflecting boundary.

(5) The strong proton acceleration caused by the reflecting boundary may be unrealistic. To construct a more realistic model, we must give up the use of a simple boundary condition (reflection or absorption) at the obstacle. A better model will include the effects of the ionospheric plasma.

Acknowledgments. For their stimulating and insightful discussions with me during the course of this work, I thank Drs. Katsuhide Marubashi (Communications Research Laboratory), Takashi Tanaka (Communications Research Laboratory), Shinobu Machida (Kyoto University), and Motohiko Tanaka (National Institute for Fusion Science).

\section{Appendix}

This appendix shows the numerical scheme used in the present simulations. The hybrid code treats ions as gyrating particles and electrons as a massless fluid. The equation of motion for an ion can be written 


$$
\begin{gathered}
m_{\mathrm{i}} \frac{d \boldsymbol{v}}{d t}=q(\boldsymbol{E}+\boldsymbol{v} / c \times \boldsymbol{B}-\eta \boldsymbol{j}), \\
\frac{d \boldsymbol{x}}{d t}=\boldsymbol{v} .
\end{gathered}
$$

Here $m_{\mathrm{i}}$ is the mass of the ion, $\boldsymbol{v}$ is its velocity, $t$ is time, $q$ is charge, $\boldsymbol{E}$ is the electric field, $c$ is the speed of light, $\boldsymbol{B}$ is the magnetic field, $\eta$ is the electric resistivity, $\boldsymbol{j}$ is the electric current density, and $\boldsymbol{x}$ is the location of the ion. The behavior of the massless electron fluid, on the other hand, is described by the equation

$$
-e\left(\boldsymbol{E}+\boldsymbol{v}_{\mathrm{e}} / c \times \boldsymbol{B}-\eta \boldsymbol{j}\right)-\frac{1}{n_{\mathrm{e}}} \nabla\left(n_{\mathrm{e}} k_{\mathrm{B}} T_{\mathrm{e}}\right)=0,
$$

where $e$ is the unit charge, $\boldsymbol{v}_{\mathrm{e}}$ and $n_{\mathrm{e}}$ are the velocity and density of the electron fluid. $T_{\mathrm{e}}$ is assumed here to be constant in space and time.

The hybrid code also solves the following two Maxwell equations:

$$
\begin{aligned}
\frac{1}{c} \frac{\partial \boldsymbol{B}}{\partial t} & =-\nabla \times \boldsymbol{E} \\
\nabla \times \boldsymbol{B} & =\frac{4 \pi}{c} \boldsymbol{j}
\end{aligned}
$$

Instead of using the Poisson equation for the electric field and charges, the hybrid code assumes a quasi-neutral condition:

$$
n_{\mathrm{e}}=n_{\mathrm{i}} \equiv n
$$

where $n_{\mathrm{i}}$ is the density of the ions. This condition excludes the electron plasma oscillation and high-frequency waves.

The simulation runs were carried out as an initial and boundary value problem. A standard leapfrog scheme was used to advance the particles and fields. We can differentiate Eq. (A.4) and get

$$
\boldsymbol{B}_{k+1}=-2 c \Delta t \nabla \times \boldsymbol{E}_{k}+\boldsymbol{B}_{k-1},
$$

where $\Delta t$ is the time step and the subscript $k$ represents the time $k \Delta t . \nabla$ is differentiated using a centered differencing scheme. Equation (A.7) is used to obtain $\boldsymbol{B}_{k+1}$ from $\boldsymbol{E}_{k}$ and $\boldsymbol{B}_{k-1}$, and then $\boldsymbol{j}_{k+1}$ is obtained from $\boldsymbol{B}_{k+1}$ by using Eq. (A.5). Differentiating Eqs. (A.1) and (A.2) yields

$$
\boldsymbol{v}_{k+1}=\left(2 q \Delta t / m_{\mathrm{i}}\right)\left(\boldsymbol{E}_{k}+\boldsymbol{v}_{k} / c \times \boldsymbol{B}_{k}-\eta \boldsymbol{j}_{k}\right)+\boldsymbol{v}_{k-1}
$$

and

$$
\boldsymbol{x}_{k+3 / 2}=\Delta t \boldsymbol{v}_{k+1}+\boldsymbol{x}_{k+1 / 2} \text {. }
$$

From these equations we can obtain the velocity and location of each ion. $\boldsymbol{B}_{k}$ and $\boldsymbol{x}_{k+1}$ are obtained using

$$
\boldsymbol{B}_{k}=(1 / 2)\left(\boldsymbol{B}_{k+1}+\boldsymbol{B}_{k-1}\right)
$$

and

$$
\boldsymbol{x}_{k+1}=(1 / 2)\left(\boldsymbol{x}_{k+3 / 2}+\boldsymbol{x}_{k+1 / 2}\right) \text {. }
$$

By summarizing ion velocities and locations, we can get the ion velocity $\boldsymbol{v}_{\mathrm{i}}$ and density $n_{\mathrm{i}}$ at each grid point. The electron velocity $\boldsymbol{v}_{\mathrm{e}}$ can be obtained from $\boldsymbol{j}, \boldsymbol{v}_{\mathrm{i}}$, and $n_{\mathrm{i}}$ :

$$
\boldsymbol{v}_{\mathrm{e}}=\left(q n_{\mathrm{i}} \boldsymbol{v}_{\mathrm{i}}-\boldsymbol{j}\right) / e n_{\mathrm{e}} .
$$

\section{References}

Alexander, C. J., J. G. Luhmann, and C. T. Russell, Interplanetary field control of the location of the Venus bow shock: Evidence for comet-like ion pickup, Geophys. Res. Lett., 13, 917-920, 1986.

Brecht, S. H., Magnetic asymmetries of unmagnetized planets, Geophys. Res. Lett., 17, 1243-1246, 1990.

Brecht, S. H. and J. R. Ferrante, Global hybrid simulation of unmagnetized planets: Comparison of Venus and Mars, J. Geophys. Res., 96, 11209_ 11220, 1991.

Brecht, S. H., J. R. Ferrante, and J. G. Luhmann, Three-dimensional simulations of the solar wind interaction with Mars, J. Geophys. Res., 98, 1345-1357, 1993

Brecht, S. H., Hybrid simulations of the magnetic topology of Mars, $J$. Geophys. Res., 102, 4743-4750, 1997.

Intriligator, D. S., Observations of mass addition to the shocked solar wind of the Venusian ionosheath, Geophys. Res. Lett., 9, 727-730, 1982.

Leroy, M. M., D. Winske, C. C. Goodrich, C. S. Wu, and K. Papadopoulos, The structure of perpendicular bow shocks, J. Geophys. Res., 87, 50815094, 1982.

Luhmann, J. G., C. T. Russell, J. R. Spreiter, and S. S. Stahara, Evidence for mass-loading of the Venus magnetosheath, Adv. Space Res., 5, 307-311, 1985.

Marubashi, K., J. M. Grebowsky, H. A. Taylor, Jr., J. G. Luhmann, C. T. Russell, and A. Barnes, Magnetic field in the wake of Venus and the formation of ionospheric holes, J. Geophys. Res., 90, 1385-1398, 1985.

Moore, K. R., V. A. Thomas, and D. J. McComas, Global hybrid simulation of the solar wind interaction with the dayside of Venus, J. Geophys. Res., 96, 7779-7791, 1991

Phillips, J. L., J. G. Luhmann, C. T. Russell, and K. R. Moore, Finite Larmor radius effect on ion pickup at Venus, J. Geophys. Res., 92, 9920-9930, 1987.

Russell, C. T., E. Chou, J. G. Luhmann, P. Gazis, L. H. Brace, and W. R. Hoegy, Solar and interplanetary control of the location of the Venus bow shock, J. Geophys. Res., 93, 5461-5469, 1988.

Saunders, M. A. and C. T. Russell, Average dimension and magnetic structure of the distant Venus magnetotail, J. Geophys. Res., 91, 5589-5604, 1986

Shimazu, H., S. Machida, and M. Tanaka, Macroparticle simulation of collisionless parallel shocks generated by solar wind and planetary plasma interactions, J. Geophys. Res., 101, 7647-7658, 1996.

Slavin, J. A. and R. E. Holzer, Solar wind flow about the terrestrial planets 1. Modeling bow shock position and shape, J. Geophys. Res., 86, 11401$11418,1981$.

Slavin, J. A., R. C. Elphic, and C. T. Russell, A comparison of Pioneer Venus and Venera bow shock observations: Evidence for a solar cycle variation, Geophys. Res. Lett., 6, 905-908, 1979

Slavin, J. A., R. E. Holzer, J. R. Spreiter, S. S. Stahara, and D. S. Chaussee, Solar wind flow about the terrestrial planets 2. Comparison with gas dynamic theory and implications for solar-planetary interactions, J. Geophys. Res., 88, 19-35, 1983.

Slavin, J. A., D. S. Intriligator, and E. J. Smith, Pioneer Venus Orbiter magnetic field and plasma observations in the Venus magnetotail, J. Geophys. Res., 94, 2383-2398, 1989.

Spreiter, J. R., A. L. Summers, and A. W. Rizzi, Solar wind flow past nonmagnetic planets-Venus and Mars, Planet. Space Sci., 18, 12811299, 1970.

Tanaka, T., Configurations of the solar wind flow and magnetic field around the planets with no magnetic field: Calculation by a new MHD simulation scheme, J. Geophys. Res., 98, 17251-17262, 1993.

Yeroshenko, Ye., W. Riedler, K. Schwingenschuh, J. G. Luhmann, M. Ong, and C. T. Russell, The magnetotail of Mars: Phobos observations, Geophys. Res. Lett., 17, 885-888, 1990.

Zhang, T. L., K. Schwingenschuh, H. Lichtenegger, W. Riedler, C. T. Russell, and J. G. Luhmann, Interplanetary magnetic field control of the Mars bow shock: Evidence for Venuslike interaction, J. Geophys. Res., 96, 11265-11269, 1991.

H. Shimazu (e-mail: shimazu@crl.go.jp)

We use Eqs. (A.3) and (A.6) to get $\boldsymbol{E}$ from $\boldsymbol{v}_{\mathrm{e}}$. 